\title{
A wait-and-reset strategy to synchronizing chaotic systems
}

\author{
Yih-Yuh Chen \\ Physics Department, National Taiwan University, Taipei, Taiwan, ROC \\ Received 11 January 1996; revised manuscript received 30 May 1996; accepted for publication 18 June 1996 \\ Communicated by A.R. Bishop
}

\begin{abstract}
A convenient way of synchronizing chaotic systems is to continuously feed one output of a driving system to the response system as one of its inputs. However, the success of this method depends crucially on the fact that the sub-Lyapunov exponents of the reduced system are negative, which may not hold for all of the system variables. We propose that this shortcoming sometimes can be overcome by not constantly monitoring the response system. We show that this somewhat counter-intuitive mechanism works because it has taken advantage of the fact that accompanying a chaotic attractor usually is a swirling motion in the phase space, which sometimes can be favorably utilized to suppress the exponential divergence typical of a chaotic system when resetting is applied regularly at a suitably chosen interval of time.
\end{abstract}

PACS: $05.45 .+\mathrm{b}$

Although the synchronization of both linear and nonlinear systems has obvious applications and has been studied by scientists and engineers for many years, the investigation of the synchronization of chaotic systems began only quite recently [1-3], but already with fruitful returns ranging from (1) the experimental realization of this idea [4-6], (2) how the originally proposed scheme might be modified and rendered more effectiveness [7], (3) how a desired behavior can be imposed on a chaotic system [8], and (4) whether chaos might be used advantageously in securely transmitting signals [9-11] to (5) its implication for spatially extended chaotic systems $[12,13]$. What is remarkable about this idea is that when synchronization is attained both the driving system and the identical response system actually are executing a chaotic motion. This seemingly impossible task has been achieved for various chaotic sys- tems, and the underlying mechanism for the synchronization is well studied by now [1]. To understand the basic idea involved, we recall that a system is chaotic because some of its associated Lyapunov exponents are positive. Yet this in no way prevents a subsystem constructed from replacing one or more of the variables of the original system by a given time-dependent signal from being Lyapunov stable. In fact, for the Lorenz equation

$$
\begin{aligned}
& \frac{\mathrm{d} X}{\mathrm{~d} t}=\sigma(-X+Y), \quad \frac{\mathrm{d} Y}{\mathrm{~d} t}=r X-Y-X Z, \\
& \frac{\mathrm{d} Z}{\mathrm{~d} t}=-b Z+X Y,
\end{aligned}
$$

it has been shown that the $Y-Z$ (or $X-Z$ ) subsystem is Lyapunov stable with respect to an identical driving system whose $X$ is shared by the response system when $\sigma=10, r=60$, and $b=\frac{8}{3}$ [1]. Because 
of this fact, it has been possible to synchronize two identical Lorenz systems by channelling the output of $X$ of one system to the other as its corresponding input, utilizing the fact that the two systems are still chaotic and thus can quickly roam through their respective phase space attractor to create chance coincidence (or more correctly, near coincidence) in the orbits even if their initial conditions are vastly different, from which point on the orbits are then quickly synchronized because the two sub-Lyapunov exponents are both negative. Synchronization using the $Y$ variable as a key can be achieved likewise. However, the same is not true with the variable $Z$ since the reduced $X-Y$ subsystem has one positive Lyapunov exponent [1], indicating that any incidental convergence of the orbits of the driving and the driven systems at one time is necessarily followed by an exponential divergence in their orbits. This naturally raises the question: does this then imply that it is impossible to synchronize the two systems using $Z$ as the driving signal?

To put things in perspective, we should note that there are two factors contributing to our inability to make the two Lorenz systems always progress side by side if $Z$ is used as the driving signal. The first one is that the Lorenz attractor has a reflective invariance with respect to the $Z$-axis, i.e., $(-X,-Y$, $Z)$ is on the attractor if the point $(X, Y, Z)$ is. This means that even if one is able to bring the two systems close enough to within a prescribed accuracy using drive $Z$, there always is the possibility that when they march in step near the neighborhood of the $Z$-axis a small fluctuation will set them off on two different but completely correlated paths $(X(t), Y(t), Z(t))$ and $(-X(t),-Y(t), Z(t))$. Whenever we talk about synchronization in this Letter, we will always leave room for this possibility. The second factor is that the direction of strong stretching of the phase flow has a major component in the $X-Y$ plane, thus making the synchronizing signal $Z$ ineffective in controlling the divergence of the orbits, a fact which is reflected in the positiveness of the largest sub-Lyapunov exponent of the $X-Y$ subsystem. It is the purpose of this Letter to offer a partial resolution to this second difficulty, which is a common feature shared by other chaotic systems. In fact, we will demonstrate that, perhaps quite contrary to one's intuition, synchronization using $Z$ as a key is

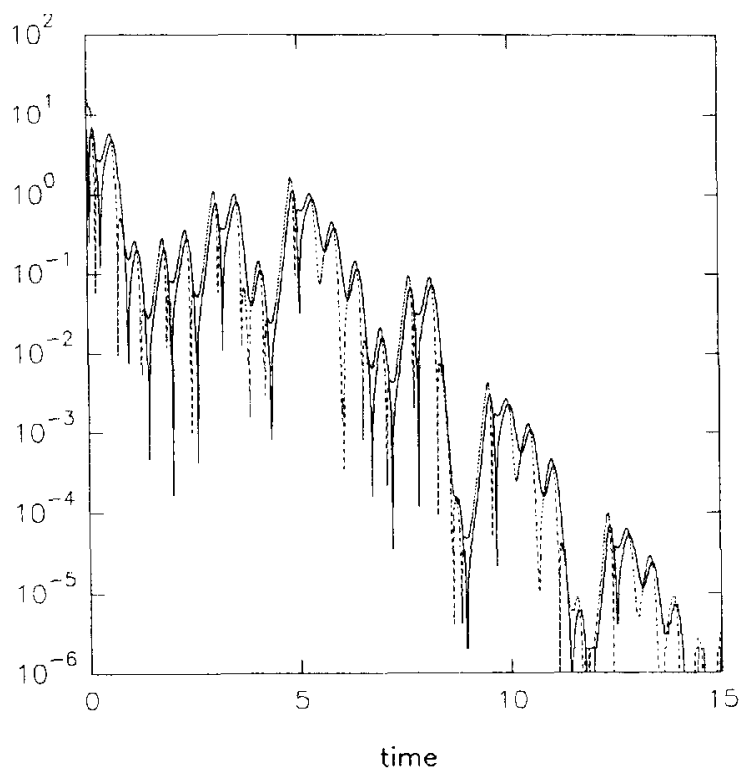

Fig. 1. The absolute values of $X^{\prime}-X$ (solid curve) and $Y^{\prime}-Y$ (dashed curve) of the Lorenz systems versus time when $Z^{\prime}$ is reset to $Z$ at a regular time interval of 0.01 .

possible if, instead of continuously monitoring the response system as has been done so far, one is willing to wait for a short while before each attempt to reset the response system.

Consider, then, a driving Lorenz system described by Eq. (1) and a response system $X^{\prime}, Y^{\prime}$ and $Z^{\prime}$ satisfying the same equation except that the last variable $\left(Z^{\prime}\right)$ is reset to $Z$ at a regular time interval $\tau$. (The traditional synchronization scheme corresponds to taking $\tau=0$.) For definiteness we will choose $\sigma=10, r=40, b=3$ and $\tau=0.01$ for this work. With the initial conditions $X=10, Y=12$, $Z=15, X^{\prime}=1, Y^{\prime}=30$ and $Z^{\prime}=2$, the absolute values of $X-X^{\prime}$ and $Y-Y^{\prime}$ are plotted in Fig. 1 as a function of time $t$. The success of synchronization is very clear from this figure. In fact, our numerical simulation shows that there is a window $0.007 \leqslant \tau \leqslant$ 0.021 in which the two systems can be synchronized.

To understand why such a synchronization scheme might work, we will first study a few simple two-dimensional examples to shed some light on the underlying physics. To begin with, let us consider the following model system

$$
\frac{\mathrm{d}}{\mathrm{d} t}\left(\begin{array}{l}
x \\
y
\end{array}\right)=\left(\begin{array}{cc}
a & \omega \\
-\omega & a
\end{array}\right)\left(\begin{array}{l}
x \\
y
\end{array}\right)
$$


which presumably approximates a certain (small) region of the phase space of some dynamical system. This equation has the solution

$x=\mathrm{e}^{a t}\left(x_{0} \cos \omega t+y_{0} \sin \omega t\right)$,

$y=\mathrm{e}^{a t}\left(y_{0} \cos \omega t-x_{0} \sin \omega t\right)$,

for given initial values $x_{0}$ and $y_{0}$. Clearly, almost all trajectories spiral out with an angular speed $\omega$ and an exponential growth rate $a$ in the radial direction, assuming that $a$ is positive. Given two identical copies of this system it is therefore impossible to synchronize them using $y$ (or $x$ ) as the drive signal because any small deviation $\delta x$ will be amplified exponentially according to $\mathrm{d} \delta x / \mathrm{d} t=a \delta x$. However, if we wait for a short time $\tau$ before resetting the $y$ variable of the response system, then $\mathrm{Eq}$. (3) predicts a deviation of

$\delta x=\delta x_{0} \mathrm{e}^{a \tau} \cos \omega \tau$,

which is seen to be always smaller than $\delta x_{0} \mathrm{e}^{a \tau}$, the amount one would obtain had we tried to synchronize them continuously. In other words, the local swirling motion in the phase space has the tendency of suppressing the exponential divergence if we wait for a short time before each reset. Because Eq. (2) is only intended to be a local approximation to the true phase flow, and because in practice two identical chaotic systems will quickly deviate in their orbits if left untended for a longer period of time, we cannot hope to obtain synchronization using this idea if $\tau$ is too large. Because of this intrinsic limitation, we will only consider the regime when $\tau$ is small here. If we then expand Eq. (5) in $\tau$ and keep up to the second order, we will obtain

$$
\frac{\delta x}{\delta x_{0}}=1+a \tau-\frac{\omega^{2}-a^{2}}{2} \tau^{2} \text {. }
$$

To be able to synchronize the two systems, we must require the expression above to have a magnitude less than one. This imposes a certain limit to which values $\tau$ can assume. Solving the inequalities, one easily derives

$$
\frac{2 a}{\omega^{2}-a^{2}}<\tau<\frac{a+\sqrt{a^{2}+4\left(\omega^{2}-a^{2}\right)}}{\omega^{2}-a^{2}},
$$

provided $\omega>a$. In order to have self-consistency, however, we must also assume that $a / \omega$ is not too large, for otherwise the reset time $\tau$ will no longer be small. This shows that, in so far as the local swirling motion (characterized by $\omega$ ) is strong enough to overcome the expansion rate (characterized by $a$ ), there is a window of reset time $\tau$ inside which synchronization can be achieved. However, we must quickly point out that the upper bound predicted by the inequality above usually represents only a rough estimate of the admissible value $\tau$ can take, because $\omega \tau$ will be of order unity when the upper bound is assumed, which will render our approximation leading to Eq. (7) a poor one.

Next, we consider, in polar coordinates, the following model equation

$$
\begin{aligned}
& \frac{\mathrm{d} r}{\mathrm{~d} t}=0, \\
& \frac{\mathrm{d} \theta}{\mathrm{d} t}=\omega+a\left(\theta-\theta_{0}\right)
\end{aligned}
$$

for some constants $\omega, a$ and $\theta_{0}$. This equation is a counterpart to Eq. (2) and is intended to model the phase flow near some point $(r, \theta)=\left(r_{0}, \theta_{0}\right)$ where the exponential divergence occurs in the same direction of the phase flow. Generally speaking, the true local phase flow of a nonlinear dynamical system will look more like some combination of Eqs. (2) and (9). Although it is possible to analyse this more general case, we would rather treat the specialized cases separately to make the physics behind as transparent as possible. With the Cartesian coordinates given by $x=r \cos \theta$ and $y=r \sin \theta$ and using $y$ as the reset variable we can easily derive the following equation for the small deviation $\delta x$,

$\frac{\delta x}{\delta x_{0}}=\cos \theta_{0} \cos \theta+\mathrm{e}^{a \tau} \sin \theta_{0} \sin \theta$,

where $\theta$ is given by

$\theta=\theta_{0}+\frac{\omega}{a}\left(\mathrm{e}^{a \tau}-1\right)$.

Again, expanding Eq. (10) in powers of the small reset time $\tau$ and keeping up to the second order, we obtain

$$
\begin{aligned}
& -\frac{\delta x}{\delta x_{0}} \\
& =1+a \tau \sin ^{2} \theta_{0} \\
& \quad-\frac{\tau^{2}}{2}\left[\omega^{2}+a^{2}\left(\cos ^{2} \theta_{0}-\frac{\omega}{a} \sin \theta_{0} \cos \theta_{0}\right)\right] .
\end{aligned}
$$


Under the assumption that $a$ is positive and is much smaller than $\omega$, this equation can be approximated by

$$
\frac{\delta x}{\delta x_{0}}=1+a \tau \sin ^{2} \theta_{0}-\frac{\omega^{2} \tau^{2}}{2} .
$$

Since the largest growth rate corresponds to $\theta_{0}=$ $\pm \pi / 2$, to be able to synchronize the response system with the driving system we must require $\tau$ to be restricted to

$$
\frac{2 a}{\omega^{2}}<\tau<\frac{2}{\omega} \text {. }
$$

Again, a window of synchronization is seen to exist for this case, which results from the fact that the strong local swirling motion is able to overcome the exponential growth coming purely from the phase space stretching. And of course, the same precaution following Eq. (7) applies to Eq. (14) as well: the upper bound calculated above might reflect an overestimate of the calculation.

Having investigated the possibility of suppressing the exponential divergence using this strategy, one must also make sure that this idea will not upset the stability of those portions of the orbit which were originally stable before the application of this technique. This corresponds to taking a negative $a$ in Eqs. (2) and (9). But in view of Eq. (5) it is quite obvious that for the model described by Eq. (2) the proposed strategy always yields a smaller value for $\left|\delta x / \delta x_{0}\right|$. Turning to the model described by Eq. (9), to get a general picture of what one can expect we may consider the limiting case when either $-a / \omega$ is very small or very large. And, with the help of either Eq. (13) or Eq. (12) we are assured that indeed the stability is retained. However, we should quickly add that there is no guarantee that the same still holds when the orders of magnitude of $a$ and $\omega$ become comparable.
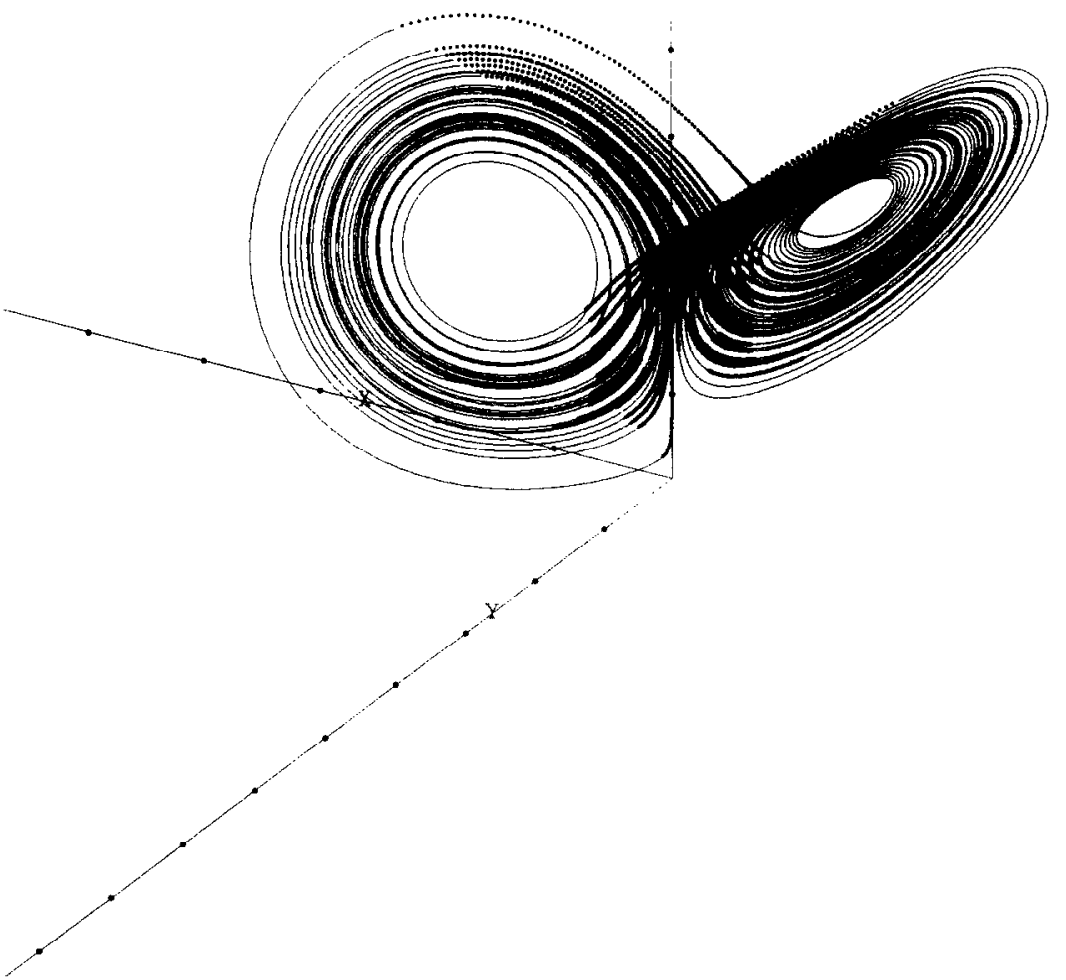

Fig. 2. A trajectory on the Lorenz attractor with points having a stretching ratio greater than that for the conventional synchronization scheme shown as small dark circles. 
To help visualize how the success of this waitand-reset strategy is made possible for the synchronization of the Lorenz equation when the reset variable is $Z$, we plot in Fig. 2 all those points along a trajectory having a growth rate $\delta x / \delta x_{0}$ of magnitude larger than that for the (failing) conventional synchronization scheme when $\tau=0.01$. From this figure, we see that, indeed, the growth rate for the majority of points on a trajectory is reduced by this method. In particular, we note that all points near the two unstable fixed points have their growth rate reduced, in accord with the experience we have gained from our analysis of Eq. (2).

Backed up by the verification from numerical simulations, we now present in the following a more systematic analysis for the most general situation. Let us write the evolution equation for the driving nonlinear dynamical system as

$$
\frac{\mathrm{d} x}{\mathrm{~d} t}=f(x)
$$

and that for the response system as

$$
\frac{\mathrm{d} x^{\prime}}{\mathrm{d} t}=f\left(x^{\prime}\right) \text {, }
$$

where some components of the variable $\boldsymbol{x}^{\prime}$ are to be reset to the values of the corresponding components of $\boldsymbol{x}$ at a regular interval of time $\tau$. Denoting the orthogonal projection onto those "free" components which are not subject to the reset by the operator $\hat{P}$ and the gradient $\nabla f$ by the operator $\hat{A}$, respectively, we see that the small deviation $\delta x \equiv x^{\prime}-x$ in the conventional synchronizing scheme $(\tau=0)$ satisfies

$\frac{\mathrm{d} \delta \boldsymbol{x}}{\mathrm{d} t}=\hat{P} \hat{A}(\delta \boldsymbol{x})$.

Correct to first order in $\delta \boldsymbol{x}$, the second order derivative in time is

$$
\frac{\mathrm{d}^{2} \delta x}{\mathrm{~d} t^{2}}=\hat{P}\left(f^{j} \frac{\partial \hat{A}}{\partial x^{j}}(\delta x)\right)+\hat{P} \hat{A} \hat{P} \hat{A}(\delta x) .
$$

Hence, correct to second order in the elapsed time $t$, we have

$$
\begin{aligned}
\delta \boldsymbol{x}_{\mathrm{c}}= & \delta \boldsymbol{x}_{0}+\hat{t \hat{P A}}\left(\delta \boldsymbol{x}_{0}\right) \\
& +\frac{t^{2}}{2}\left[\hat{P}\left(f^{j} \frac{\partial \hat{A}}{\partial x^{j}}\left(\delta x_{0}\right)\right)+\hat{P} \hat{A} \hat{P} \hat{A}\left(\delta x_{0}\right)\right],
\end{aligned}
$$

with $\delta x_{0}$ being the deviation right after the previous reset, the subscript $c$ meaning it is for the case when the reset is performed continuously, and with an implied summation convention on the index $j$. Recalling that in our wait-and-reset approach the deviation $\delta x_{w}$ is allowed to freely evolve according to

$\frac{\mathrm{d} \delta \boldsymbol{x}_{\mathrm{w}}}{\mathrm{d} t}=\hat{A}(\delta x)$

between two successive resets, we can repeat the same calculation and derive

$$
\begin{aligned}
\delta x_{\mathrm{w}}= & \delta x_{0}+t \hat{P} \hat{A}\left(\delta x_{0}\right) \\
& +\frac{t^{2}}{2}\left[\hat{P}\left(f^{j} \frac{\partial \hat{A}}{\partial x^{j}}\left(\delta x_{0}\right)\right)+\hat{P} \hat{A A}\left(\delta x_{0}\right)\right] .
\end{aligned}
$$

Calculating the squared norm of the vector $\delta x_{\mathrm{c}}$ and $\delta \boldsymbol{x}_{\mathrm{w}}$ after time $t=\tau$ we therefore obtain

$$
\begin{aligned}
\left\|\delta \boldsymbol{x}_{\mathrm{w}}\right\|^{2}= & \left\|\delta \boldsymbol{x}_{\mathrm{c}}\right\|^{2} \\
& +\tau^{2}\left\langle\delta \boldsymbol{x}_{0} \mid(\hat{P} \hat{A} \hat{A}-\hat{P} \hat{A} \hat{P} \hat{A}) \delta x_{0}\right\rangle,
\end{aligned}
$$

where $\langle\cdots \mid \cdots\rangle$ is the Dirac notation for inner product of two vectors, and we have retained only terms up to second order in $\tau$. With $\hat{P}^{\perp} \equiv$ Identity $-\hat{P}$ and noting that the projection of $\delta x_{0}$ under $\hat{P}$ remains the same, we can recast this equation to read

$\left\|\delta x_{\mathrm{w}}\right\|^{2}=\left\|\delta \boldsymbol{x}_{\mathrm{c}}\right\|^{2}+\tau^{2}\left\langle\delta x_{0} \mid\left(\hat{A} \hat{P}^{\perp} \hat{A}\right) \delta x_{0}\right\rangle$.

The geometrical interpretation of the second term in Eq. (23) is rather simple: it measures how the initial deviation $\delta x_{0}$ curls up into those directions that are to be reset after time $\tau$ (thus the appearance of $\hat{P} \perp \hat{A} \delta x_{0}$ ), and how those components subsequently evolve to curl back to contribute a component in the space of free variables again (thus the follow-up operation by $\left.\left\langle\delta \boldsymbol{x}_{0}\right| \hat{\boldsymbol{A}}\right)$. From this, one sees that whenever our strategy works it is because the local (relative) swirling motion has curled back to suppress the otherwise exponential stretching. Our success of this strategy with the Lorenz equation therefore suggests that this local effect can be favorably exploited to actually stabilize the synchronization that was previously impossible. 
Lest the reader gets the impression from the motivating examples that the wait-and-reset approach has a tendency of suppressing the exponential divergence as compared to the continuous reset scheme only when the relative local phase flow is of

(a)

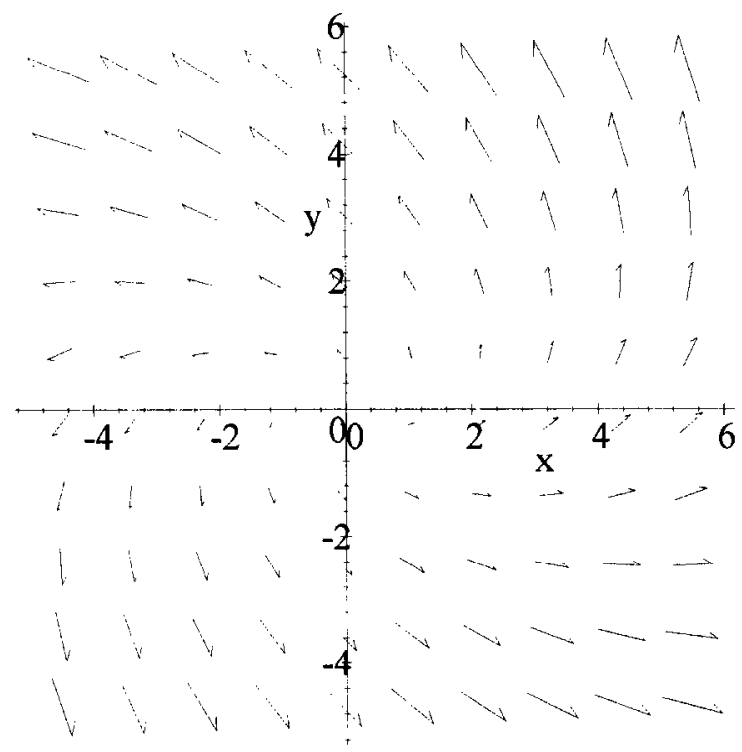

(c)

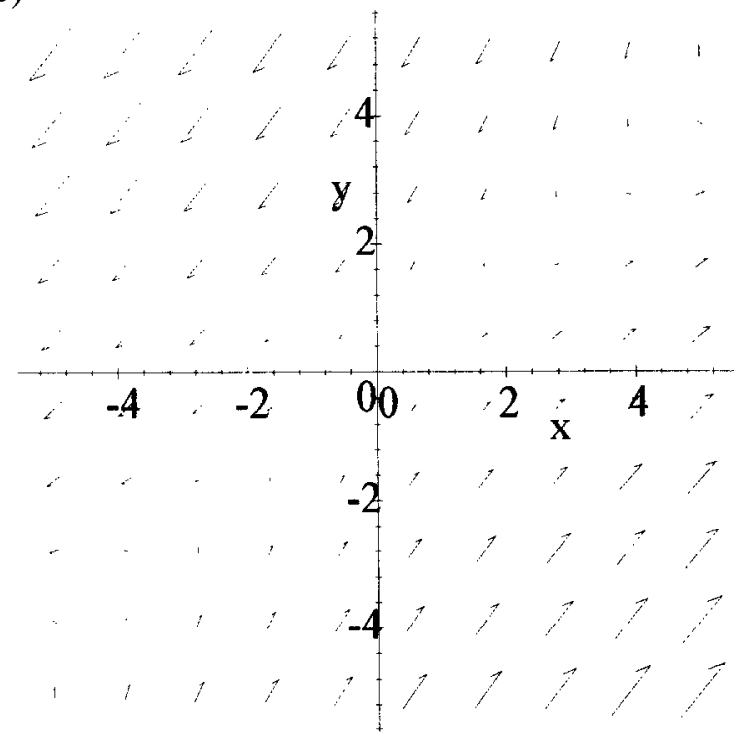

the spiral type, we should like to point out that one can use Eq. (23) as a guideline to sharpen the as yet undefined concept of "favorable swirling motion". For instance, if we arrange the variables so that the first $n$ of them are the free variables and the remain-

(b)

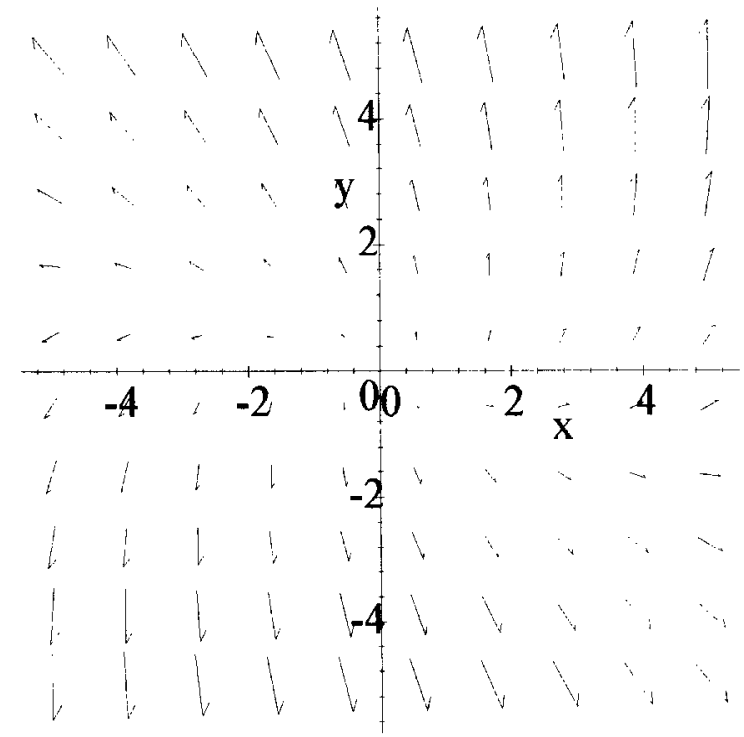

(d)

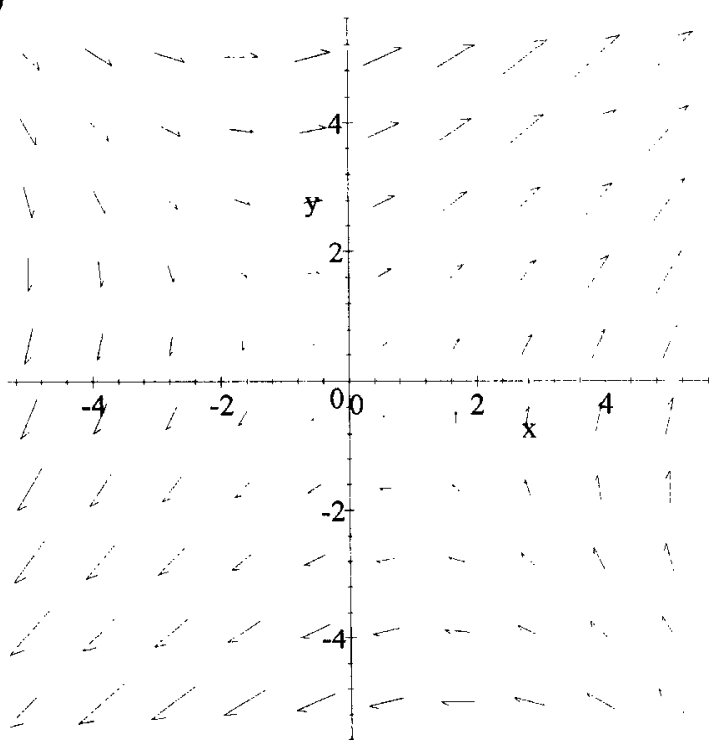

Fig. 3. Different phase portraits for Eq. (26) demonstrating geometrically why and when wait-and-reset works. (a)-(c) correspond to the three cases (spiral, expanding, and saddle) depicted in Eq. (27) and (d) is the saddle described by Eq. (28) for which wait-and-reset should not be applied. 
ing $m$ variables are subject to reset, then we may write

$\hat{P} \equiv\left(\begin{array}{cc}I_{n} & 0 \\ 0 & 0\end{array}\right), \quad \hat{P}^{\perp} \equiv\left(\begin{array}{cc}0 & 0 \\ 0 & I_{m}\end{array}\right), \quad \hat{A} \equiv\left(\begin{array}{ll}a & c \\ b & d\end{array}\right)$,

where $a, b, c$ and $d$ are matrices of dimension $n \times n, m \times n, n \times m$, and $m \times m$, respectively, and

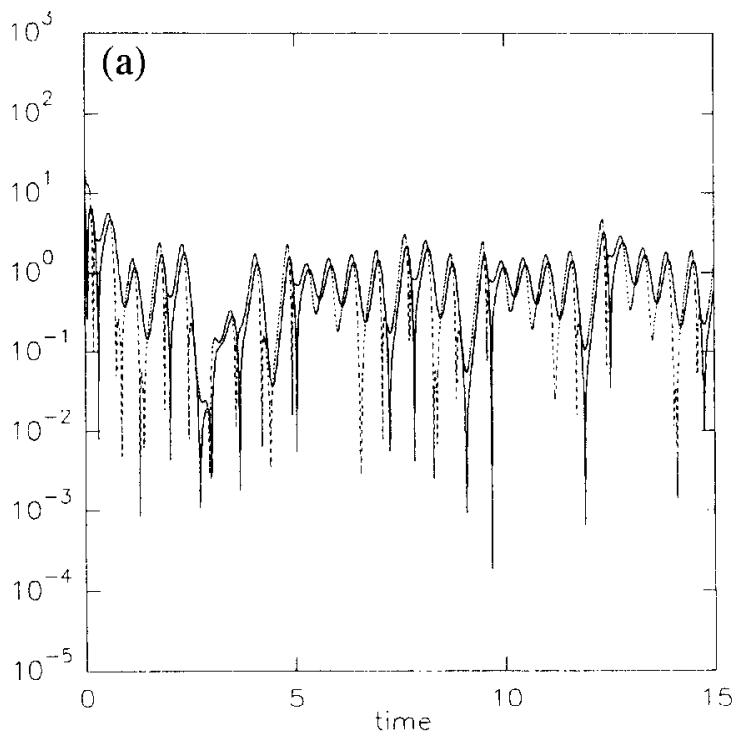

$I_{i}$ is the $i \times i$ identity matrix. This allows one to simplify the second term of Eq. (23), which now reads

$\tau^{2}\left\langle\delta x_{0}|c b| \delta x_{0}\right\rangle$

For the special case when $m=n=1$ this term is negative as long as both the $x$-and the $y$-axes are tilted in the same sense under the phase flow after a

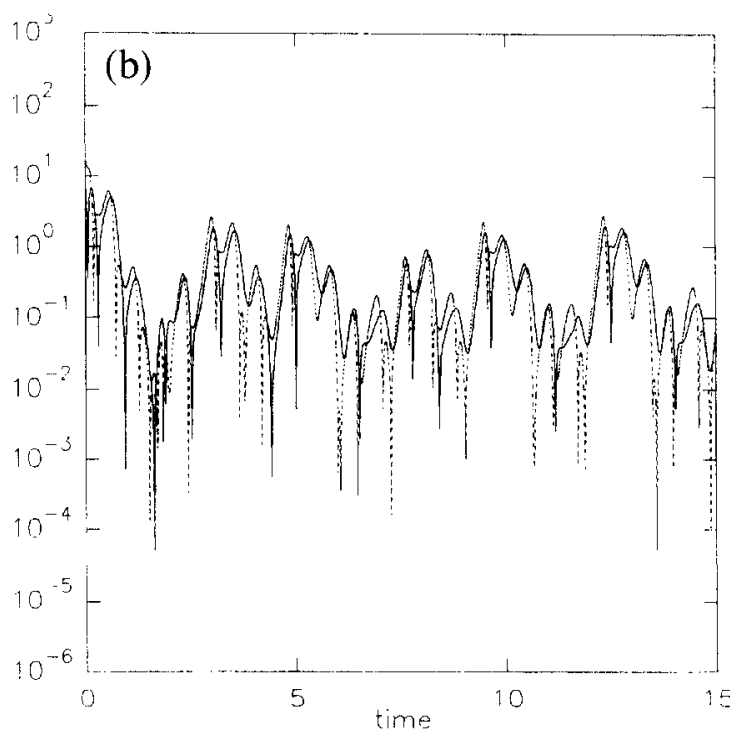

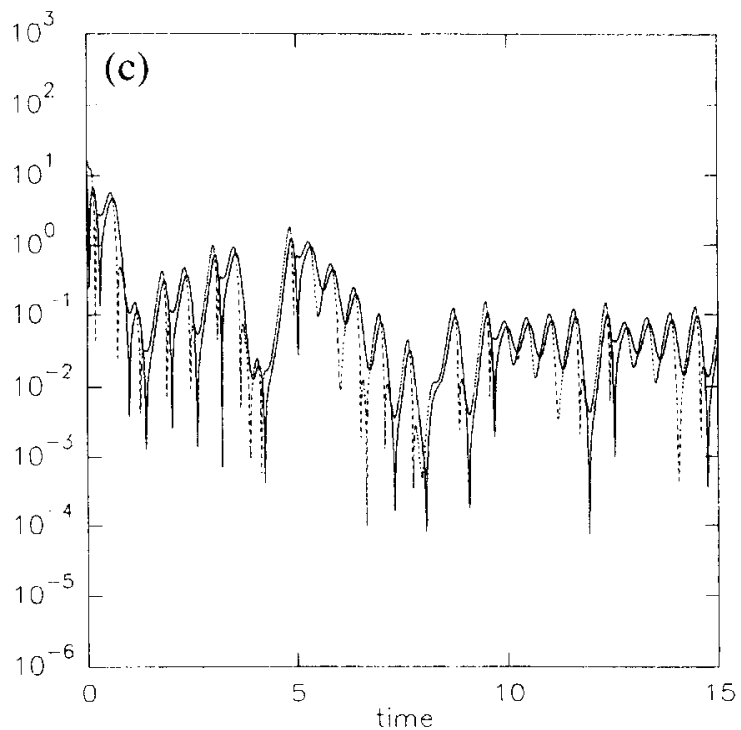

Fig. 4. $\left|X^{\prime}-X\right|$ (solid curve) and $\left|Y^{\prime}-Y\right|$ (dashed curve) versus time when the only mismatching pamameter is (a) $r^{\prime}=40.1$, (b) $\sigma^{\prime}=10.1$, and (c) $b^{\prime}=3.03$, respectively. 
small time $\tau$. Thus, the swirling motion is favorable for the linear system

$\frac{\mathrm{d}}{\mathrm{d} t}\left(\begin{array}{l}\delta x \\ \delta y\end{array}\right)=\hat{A}\left(\begin{array}{l}\delta x \\ \delta y\end{array}\right)$

if $\hat{A}$ is, say,

$\left(\begin{array}{cc}1 & -2 \\ 2 & 1\end{array}\right)$ (the phase flow spirals outward), or

$\left(\begin{array}{cc}2 & -2 \\ 2 & 7\end{array}\right)$ (the phase flow has two expanding

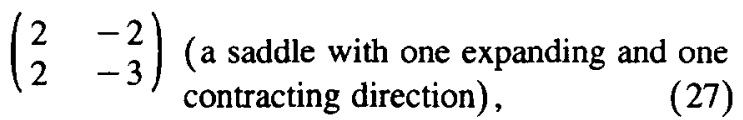

though in the last case the suppression is not strong enough to overcome the original exponential divergence. In fact, quite often a glance of the phase space portrait readily tells us if the wait-and-reset strategy can be adopted to one's advantage for synchronization, as illustrated in Fig. 3. (The basic idea cannot be simpler: if a trajectory starting on the $x$-axis tends to cross the $y$-axis at some later time, then wait-and-reset works fine for a suitably chosen т.) Of course, this analysis also tells us when the wait-and-reset strategy is expected not to work as we would like it to: this can happen, for example, when the coordinate axes tend to align themselves under evolution, as exemplified in

$\hat{A}=\left(\begin{array}{ll}1 & 3 \\ 3 & 1\end{array}\right) \quad$ (a saddle)

(see Fig. 3). However, because the chaotic attractor is usually finite in extent, the flow on the attractor necessarily exhibits a lot of swirling motion, and it seems plausible that the method we report in this Letter might be of use to many systems.

If it is desired to have a more quantitative measure of whether two identical chaotic systems can be brought to synchronization using the proposed idea, one can define a time- $\tau$ Lyapunov exponent $\lambda_{\tau}$ in the following way. First, we note that Eq. (20) defines a linear operator $\hat{D}_{1}$ mapping $\delta x_{0}$ to $\delta \boldsymbol{x}_{\mathrm{w}}(t=\tau)$. Similarly, for the following pairs of consecutive resets we have a series of operators $\hat{D}_{2}, \hat{D}_{3}, \ldots$. From this knowledge we may com- pound the operations to define $\lambda_{\tau}$ as the eigenvalues of

$\lim _{N \rightarrow \infty} \frac{1}{2 N \tau} \ln \left(\hat{D}_{1}^{\dagger} \ldots \hat{D}_{N}^{\dagger} \hat{D}_{N} \ldots \hat{D}_{1}\right)$,

which is merely a generalization of the familiar Lyapunov exponents [14]. Then, a necessary condition that our scheme works is that the largest of the $\lambda_{\tau}$ 's be negative. Taking a time step of $10^{-4}$ and integrating the Lorenz equation for a total time span of 100 , we have visually tracked the orbits to confirm that they are in fact not synchronized and have computed the largest $\lambda_{\tau}$ to be +0.02 when $\tau$ is 0 ; and for the case when $\tau=0.01$ our calculation gives $\lambda_{\tau}=-0.24$, indicating that $\lambda_{\tau}$ can be a useful measure of whether synchronization is to be expected.

We have also checked if a slight parameter mismatch might disrupt the synchronization. This is shown in Fig. 4 for the cases when the only mismatching parameter of the response system is respectively chosen to be (a) $r=40.1$, (b) $\sigma=10.1$, and (c) $b=3.03$. As one might have expected, the per-

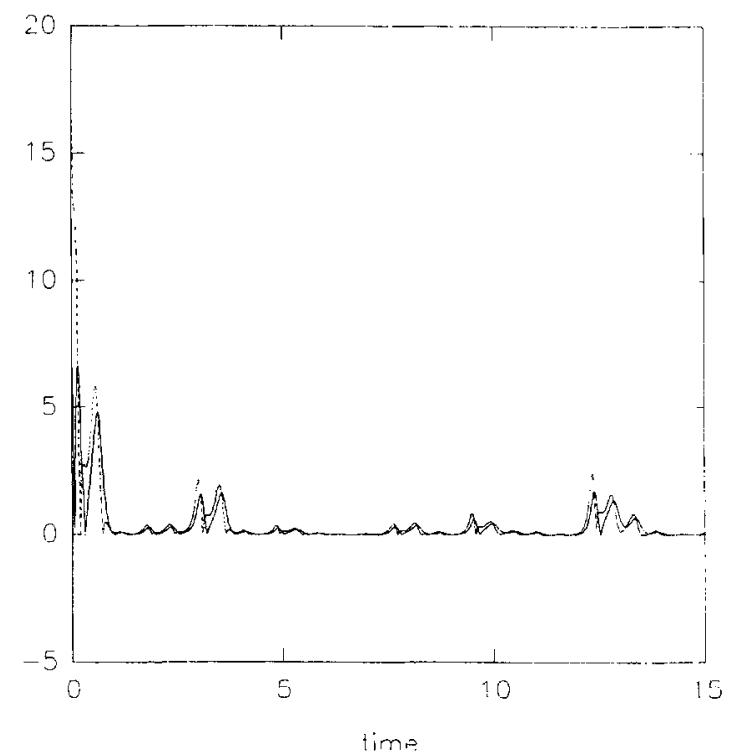

Fig. 5. The smaller of $\left|X^{\prime}-X\right|\left(\left|Y^{\prime}-Y\right|\right)$ and $\left|X^{\prime}+X\right|\left(\mid Y^{\prime}\right.$ $+Y \mid)$ is plotted in the solid (dashed) curve as a function of time when a noise of amplitude 0.1 is added on to the driving signal $Z$ before it is fed into the response system. The peaks between two adjacent synchronized configurations signify a rapid flip of the response system from one state to the other equally admissible symmetrical state. 
turbation caused by a small mismatch in any of the three parameters is only a fluctuation superimposed on the synchronized state. (In terms of percentage, the safe margin of mismatch for $r$ within which synchronization can be maintained is found to be the smallest among all the three parameters of the Lorenz equation.) Likewise, we have also checked that a small noise added upon the resetting signal $Z$ before it is fed into the response system produces a similar fluctuating part to the otherwise synchronized state. This is shown in Fig. 5 for a noise of an amplitude of 0.1 . Note that the sporadic peaks in this figure correspond to the orbits being close to the $Z$-axis and thus making the driving signal $Z$ incapable of doing its job effectively. In fact, our numerical simulations show that the phenomenon is similarly conspicuous when the mismatch in the system parameters is not very small.

To summarize, we have demonstrated that synchronization of chaotic systems might still be possible even if the reduced system has a positive subLyapunov exponent. In order to achieve synchronization under such a circumstance, we have suggested that the wait-and-reset strategy be used with a suitable waiting time $\tau$. This method works because the swirling phase flow present on many chaotic attractors can be taken advantage of to suppress the exponential divergence, even if the divergence occurs along the direction of the phase flow. The synchronization is shown to be robust with respect to small noises and parameter mismatches in the response system. Because the underlying principle of this method has a simple geometrical origin, it is expected to work for many systems. The usefulness of this idea in practical applications, however, remains to be investigated.

This work was supported by the National Science Council of the Republic of China under grant number NSC85-2112-M002-010 and the Gou-Pee Papers Foundation.

\section{References}

[1] L.M. Pecora and T.L. Carroll, Phys. Rev. Lett. 64 (1990) 821.

[2] T.L. Carroll and L.M. Pecora, Physica D 67 (1993) 126.

[3] R. He and P.G. Vaidya, Phys. Rev. A 46 (1992) 7387.

[4] E.R. Hunt, Phys. Rev. Lett. 67 (1991) 1953.

[5] T.C. Newell, P.M. Alsing, A. Gavrielides and V. Kovanis, Phys. Rev. Lett. 72 (1994) 1647.

[6] R. Roy and K.S. Thornburg Jr., Phys. Rev. Lett. 72 (1994) 2009.

[7] M. Ding and E. Ott, Phys. Rev. E 49 (1994) R945.

[8] M.A. Matías and J. Güemez, Phys. Lett. A 209 (1995) 48.

[9] S. Hayes, C. Grebogi, E. Ott and A. Mark, Phys. Rev. Lett. 73 (1994) 1781.

[10] N. Gershenfeld and G. Grinstein, Phys. Rev. Lett. 74 (1995) 5024.

[11] L. Kocarev and U. Parlitz, Phys. Rev. Lett. 74 (1995) 5028.

[12] H.G. Winful and L. Rahman, Phys. Rev. Lett. 65 (1990) 1575.

[13] J.F. Heagy, T.L. Carroll and L.M. Pecora, Phys. Rev. E 50 (1994) 1874.

[14] E. Ott, Chaos in dynamical systems (Cambridge Univ. Press, Cambridge. 1993). 